\title{
The magnetic field at milliarcsecond resolution around IRAS 20126+4104
}

\author{
G. Surcis ${ }^{1}$, W. H. T. Vlemmings ${ }^{2}$, H. J. van Langevelde ${ }^{1,3}$, L. Moscadelli ${ }^{4}$, and B. Hutawarakorn Kramer ${ }^{5,6}$ \\ 1 Joint Institute for VLBI in Europe, Postbus 2, 79990 AA Dwingeloo, The Netherlands \\ 2 Chalmers University of Technology, Onsala Space Observatory, 43992 Onsala, Sweden \\ 3 Sterrewacht Leiden, Leiden University, Postbus 9513, 2300 RA Leiden, The Netherlands \\ 4 INAF - Osservatorio Astrofisico di Arcetri, Largo E. Fermi 5, 50125 Firenze, Italy \\ 5 Max-Planck Institut für Radioastronomie, Auf dem Hügel 69, 53121 Bonn, Germany \\ ${ }^{6}$ National Astronomical Research Institute of Thailand, Ministry of Science and Technology, Rama VI Rd., 10400 Bangkok, Thailand
}

Received 4 October 2013 / Accepted 20 January 2014

\section{ABSTRACT}

\begin{abstract}
Context. IRAS $20126+4104$ is a well studied B0.5 protostar that is surrounded by a $\sim 1000$ au Keplerian disk and is where a largescale outflow originates. Both 6.7- $\mathrm{GHz} \mathrm{CH}_{3} \mathrm{OH}$ masers and 22- $\mathrm{GHz} \mathrm{H}_{2} \mathrm{O}$ masers have been detected toward this young stellar object. The $\mathrm{CH}_{3} \mathrm{OH}$ masers trace the Keplerian disk, while the $\mathrm{H}_{2} \mathrm{O}$ masers are associated with the surface of the conical jet. Recently, observations of dust polarized emission $(350 \mu \mathrm{m})$ at an angular resolution of $9 \operatorname{arcsec}(\sim 15000 \mathrm{au})$ have revealed an $S$-shaped morphology of the magnetic field around IRAS $20126+4104$.

Aims. The observations of polarized maser emissions at milliarcsecond resolution ( $20 \mathrm{au})$ can make a crucial contribution to understanding the orientation of the magnetic field close to IRAS 20126+4104. This will allow us to determine whether the magnetic field morphology changes from arcsecond resolution to milliarcsecond resolution.

Methods. The European VLBI Network was used to measure the linear polarization and the Zeeman splitting of the 6.7-GHz $\mathrm{CH}_{3} \mathrm{OH}$ masers toward IRAS 20126+4104. The NRAO Very Long Baseline Array was used to measure the linear polarization and the Zeeman splitting of the 22- $\mathrm{GHz} \mathrm{H}_{2} \mathrm{O}$ masers toward the same region.

Results. We detected $26 \mathrm{CH}_{3} \mathrm{OH}$ masers and $5 \mathrm{H}_{2} \mathrm{O}$ masers at high angular resolution. Linear polarization emission was observed toward three $\mathrm{CH}_{3} \mathrm{OH}$ masers and toward one $\mathrm{H}_{2} \mathrm{O}$ maser. Significant Zeeman splitting was measured in one $\mathrm{CH}_{3} \mathrm{OH}$ maser $\left(\Delta V_{\mathrm{Z}}=-9.2 \pm 1.4 \mathrm{~m} \mathrm{~s}^{-1}\right)$. No significant $(5 \sigma)$ magnetic field strength was measured using the $\mathrm{H}_{2} \mathrm{O}$ masers. We found that in IRAS 20126+4104 the rotational energy is less than the magnetic energy.
\end{abstract}

Key words. stars: formation - masers - polarization - magnetic fields - ISM: individual objects: IRAS 20126+4104

\section{Introduction}

In the past years, the formation of high-mass stars has been at the center of numerous studies, both observational and theoretical. The observations reveal that the structure of massive protostars is probably similar to that of their less massive counterpart (e.g., Tang et al. 2009; Keto \& Zhang 2010; Johnston et al. 2013), and the theoretical simulations match the observations as long as the magnetic field is taken into consideration (e.g., Peters et al. 2011; Seifried et al. 2012a; Myers et al. 2013).

One of the typical characteristics of low-mass protostars that has also been observed around high-mass protostars (B-type stars) is the presence of circumstellar disks (e.g., Cesaroni et al. 2006, 2007). Seifried et al. (2011) show that Keplerian disks with sizes of a few 100 au are easily formed around massive protostars when a weak magnetic field is considered in the simulations. The Keplerian disks are also formed if a strong magnetic field is present but only if a turbulent velocity field is introduced (Seifried et al. 2012b).

Determining the morphology of magnetic fields close to circumstellar disks or tori in the early stages of massive star formation is very difficult mainly because the massive protostars are distant, rare, and quick to evolve. However, it was possible in some cases, for instance in Cepheus A (Vlemmings et al. 2010) and in NGC 7538 (Surcis et al. 2011a), where the 6.7-GHz $\mathrm{CH}_{3} \mathrm{OH}$ maser emission was used to probe the magnetic field at milliarcsecond (mas) resolution (i.e., $\sim 10 \mathrm{au}$ ). In both cases, the masers trace the infalling gas but not the disk/torus material directly. A suitable case where the magnetic field can be measured on the surface of a disk may instead be IRAS $20126+4104$.

IRAS $20126+4104$ is a well studied B0.5 protostar $(M=$ $7 M_{\odot}$ ) at a distance of $1.64 \pm 0.05 \mathrm{kpc}$ (Moscadelli et al. 2011, hereafter MCR11). A disk of $\sim 1000$ au $\left(\mathrm{PA}_{\text {disk }}=53^{\circ} \pm 7^{\circ}\right.$, Cesaroni et al. 2005), which is undergoing Keplerian rotation, was imaged by Cesaroni et al. (1997, 1999, 2005). In addition, a jet/outflow perpendicular to the disk $\left(\mathrm{PA}_{\text {jet }}=115^{\circ}\right.$, MCR11), which shows a precession motion around the rotation axis of the disk (e.g., Shepherd et al. 2000), was also detected from small- $\left(\sim 10^{2} \mathrm{au}\right)$ to large-scale $\left(\sim 10^{4}\right.$ au) (e.g., Cesaroni et al. 1997, 1999, 2013; Hofner et al. 2007; Caratti o Garatti 2008; MCR11). The three maser species 6.7- $\mathrm{GHz} \mathrm{CH}_{3} \mathrm{OH}$, 1.6- $\mathrm{GHz} \mathrm{OH}$, and 22- $\mathrm{GHz} \mathrm{H}_{2} \mathrm{O}$ were detected (Edris et a. 2005; Moscadelli et al. 2005; MCR11). The former can be divided into two groups, i.e. Groups 1 and 2. While Group 1 is associated to the Keplerian disk, Group 2 shows relative proper motions, indicating that the masers are moving perpendicularly away from the disk (MCR11). The $\mathrm{OH}$ masers have an elongated distribution and trace part of the Keplerian disk (Edris et al. 2005). Edris et al. (2005) also identified one Zeeman pair of $\mathrm{OH}$ masers that indicates a magnetic field strength of $\sim+11 \mathrm{mG}$. The $\mathrm{H}_{2} \mathrm{O}$ masers are instead associated with the surface of the 
A\&A 563, A30 (2014)

Table 1. Parameters of the 6.7- $-\mathrm{GHz} \mathrm{CH}_{3} \mathrm{OH}$ maser features detected in IRAS $20126+4104$.

\begin{tabular}{|c|c|c|c|c|c|c|c|c|c|c|c|c|c|}
\hline $\begin{array}{c}(1) \\
\text { Maser }\end{array}$ & $\begin{array}{c}(2) \\
\text { Group }\end{array}$ & $\begin{array}{c}\text { (3) } \\
\mathrm{RA}^{a} \\
\text { offset } \\
\text { (mas) }\end{array}$ & $\begin{array}{c}(4) \\
\text { Dec }^{a} \\
\text { offset } \\
\text { (mas) }\end{array}$ & $\begin{array}{c}\text { (5) } \\
\text { Peak flux } \\
\text { density(I) } \\
\text { (Jy/beam) }\end{array}$ & $\begin{array}{c}(6) \\
V_{\mathrm{lsr}} \\
\left(\mathrm{km} \mathrm{s}^{-1}\right) \\
\end{array}$ & $\begin{array}{c}(7) \\
\Delta v_{\mathrm{L}} \\
\left(\mathrm{km} \mathrm{s}^{-1}\right)\end{array}$ & $\begin{array}{l}(8) \\
P_{1}^{b} \\
(\%) \\
\end{array}$ & $\begin{array}{l}(9) \\
\chi^{b} \\
\left({ }^{\circ}\right) \\
\end{array}$ & $\begin{array}{c}(10) \\
\Delta V_{\mathrm{i}}{ }^{c} \\
\left(\mathrm{~km} \mathrm{~s}^{-1}\right)\end{array}$ & $\begin{array}{c}(11) \\
T_{B} \Delta \Omega^{c} \\
(\log \mathrm{K} \text { sr) }\end{array}$ & $\begin{array}{l}(12) \\
P_{\mathrm{V}} \\
(\%) \\
\end{array}$ & $\begin{array}{c}(13) \\
\Delta V_{\mathrm{Z}} \\
\left(\mathrm{m} \mathrm{s}^{-1}\right) \\
\end{array}$ & $\begin{array}{c}(14) \\
\theta^{d} \\
\left({ }^{\circ}\right) \\
\end{array}$ \\
\hline M01 & 2 & -14.869 & 5.734 & $0.917 \pm 0.003$ & -6.72 & 0.29 & - & - & - & - & - & - & - \\
\hline M02 & 2 & -11.405 & 3.063 & $0.964 \pm 0.003$ & -6.72 & 0.36 & - & - & - & - & - & - & - \\
\hline M03 & 2 & -2.797 & 19.127 & $0.265 \pm 0.009$ & -6.10 & 0.20 & - & - & - & - & - & - & - \\
\hline M04 & 2 & -1.743 & -16.438 & $0.275 \pm 0.007$ & -5.97 & 0.25 & - & - & - & - & - & - & - \\
\hline M05 & 2 & 0 & 0 & $27.838 \pm 0.009$ & -6.10 & 0.36 & $1.6 \pm 0.4$ & $-65 \pm 3$ & $2.0_{-0.2}^{+0.1}$ & $8.8_{-0.4}^{+0.8}$ & 0.6 & $-9.2 \pm 1.4$ & $75_{-43}^{+10}$ \\
\hline M06 & 2 & 0.129 & -7.450 & $0.316 \pm 0.007$ & -6.14 & 0.30 & - & - & -0.2 & -0.4 & - & - & - \\
\hline M07 & 2 & 0.947 & 8.122 & $0.543 \pm 0.008$ & -6.01 & 0.31 & - & - & - & - & - & - & - \\
\hline M08 & 2 & 9.382 & -4.685 & $0.428 \pm 0.007$ & -5.97 & 0.25 & - & - & - & - & - & - & - \\
\hline M09 & 2 & 19.883 & -7.031 & $0.199 \pm 0.007$ & -6.23 & 0.20 & - & - & - & - & - & - & - \\
\hline M10 & 2 & 19.904 & -11.261 & $0.049 \pm 0.003$ & -5.66 & 0.23 & - & - & - & - & - & - & - \\
\hline M11 & 2 & 52.634 & -48.145 & $0.068 \pm 0.002$ & -5.13 & 0.28 & - & - & - & - & - & - & - \\
\hline M12 & 2 & 56.313 & -21.915 & $0.308 \pm 0.002$ & -5.57 & 0.38 & - & - & - & - & - & - & - \\
\hline M13 & 2 & 62.231 & -15.331 & $0.861 \pm 0.003$ & -6.41 & 0.27 & - & - & - & - & - & - & - \\
\hline M14 & 2 & 81.877 & -10.986 & $0.919 \pm 0.003$ & -6.67 & 0.28 & - & - & - & - & - & - & - \\
\hline M15 & & 83.835 & 244.766 & $0.070 \pm 0.003$ & -6.50 & 0.19 & - & - & - & - & - & - & - \\
\hline M16 & 1 & 166.917 & -77.072 & $0.048 \pm 0.002$ & -5.18 & 0.19 & - & - & - & - & - & - & - \\
\hline M17 & 1 & 155.448 & -104.588 & $0.178 \pm 0.002$ & -4.87 & 0.23 & - & - & - & - & - & - & - \\
\hline M18 & 1 & 191.556 & 37.796 & $1.851 \pm 0.003$ & -7.64 & 0.27 & $1.4 \pm 0.1$ & $-71 \pm 5$ & $1.4_{-0.2}^{+0.2}$ & $8.8_{-0.1}^{+0.3}$ & - & - & $85_{-34}^{+6}$ \\
\hline M19 & 1 & 192.782 & 25.593 & $0.117 \pm 0.003$ & -7.68 & 0.23 & - & - & -0.2 & -0.1 & - & - & -34 \\
\hline M20 & 1 & 204.295 & 7.683 & $0.309 \pm 0.003$ & -7.11 & 0.21 & - & - & - & - & - & - & - \\
\hline M21 & 1 & 207.436 & 33.264 & $0.072 \pm 0.003$ & -7.51 & 0.19 & - & - & - & - & - & - & - \\
\hline M22 & 1 & 210.965 & 16.399 & $0.114 \pm 0.003$ & -6.50 & 0.21 & - & - & - & - & - & - & - \\
\hline M23 & 1 & 215.312 & 8.267 & $0.402 \pm 0.003$ & -6.98 & 0.36 & - & - & - & - & - & - & - \\
\hline M24 & 1 & 237.153 & 4.383 & $2.154 \pm 0.003$ & -6.98 & 0.30 & $0.6 \pm 0.2$ & $56 \pm 43$ & $1.6_{-02}^{+0.2}$ & $8.4_{-0.4}^{+0.4}$ & - & - & $79_{-37}^{+11}$ \\
\hline M25 & 1 & 261.232 & 4.707 & $0.166 \pm 0.003$ & -7.72 & 0.28 & - & - & -0.2 & -0.4 & - & - & - \\
\hline M26 & 1 & 277.392 & 3.834 & $0.644 \pm 0.002$ & -8.25 & 0.26 & - & - & - & - & - & - & - \\
\hline
\end{tabular}

Notes. ${ }^{(a)}$ The reference position is $\alpha_{2000}=20^{\mathrm{h}} 14^{\mathrm{m}} 26^{\mathrm{s}} .046 \pm 0^{\mathrm{s}} .001$ and $\delta_{2000}=41^{\circ} 13^{\prime} 32^{\prime \prime} .690 \pm 0^{\prime \prime} .009$ (see Sect. 4). ${ }^{(b)} P_{1}$ and $\chi$ are the mean values of the linear polarization fraction and the linear polarization angle measured across the spectrum, respectively. ${ }^{(c)}$ The best-fitting results obtained by using a model based on the radiative transfer theory of methanol masers for $\Gamma+\Gamma_{v}=1 \mathrm{~s}^{-1}$ (Vlemmings et al. 2010; Surcis et al. 2011a). The errors were determined by analyzing the full probability distribution function. ${ }^{(d)}$ The angle between the magnetic field and the maser propagation direction is determined by using the observed $P_{1}$ and the fitted emerging brightness temperature. The errors were determined by analyzing the full probability distribution function.

conical jet (opening angle $=9^{\circ}$ ), with speed increasing for increasing distance from the protostar (Moscadelli et al. 2005; MCR11).

Shinnaga et al. (2012) measured the polarized dust emission at $350 \mu \mathrm{m}$ at arcsec resolution $\left(\sim 10^{4}\right.$ au $)$ by using the SHARC II Polarimeter (SHARP) with the 10.4 m Leighton telescope at the Caltech Submillimeter Observatory (CSO). They determined that the global magnetic field is oriented north-south, but it changes its direction close to the protostar becoming parallel to the Keplerian disk; i.e., here the field is nearly perpendicular to the rotation axis of the disk. The apparent jet precession could be explained by the misalignment of the magnetic field and the rotation axis (Shinnaga et al. 2012).

The observations of polarized emissions of $6.7-\mathrm{GHz}$ $\mathrm{CH}_{3} \mathrm{OH}$ and $22-\mathrm{GHz} \mathrm{H}_{2} \mathrm{O}$ masers offer a possibility to better determine the morphology of the magnetic field close to the circumstellar disk and to the jet. For this reason, here we present both European VLBI Network (EVN) observations of $\mathrm{CH}_{3} \mathrm{OH}$ masers and Very Long Baseline Array (VLBA) observations of $\mathrm{H}_{2} \mathrm{O}$ masers that were carried on in full polarization mode.

\section{Observations}

\subsection{7-GHz EVN data}

IRAS $20126+4104$ was observed at $6.7-\mathrm{GHz}$ in full polarization spectral mode with seven of the EVN ${ }^{1}$ antennas (Effelsberg,

\footnotetext{
1 The European VLBI Network is a joint facility of European, Chinese, South African, and other radio astronomy institutes funded by their national research councils.
}

Jodrell, Onsala, Medicina, Torun, Westerbork, and Yebes-40 m), for a total observation time of $5.5 \mathrm{~h}$, on October 30, 2011 (program code ES066). The bandwidth was $2 \mathrm{MHz}$, providing a velocity range of $\sim 100 \mathrm{~km} \mathrm{~s}^{-1}$. The data were correlated with the EVN software correlator (SFXC) at the Joint Institute for VLBI in Europe (JIVE) using 2048 channels and generating all four polarization combinations (RR, LL, RL, LR) with a spectral resolution of $\sim 1 \mathrm{kHz}\left(\sim 0.05 \mathrm{~km} \mathrm{~s}^{-1}\right)$.

The data were edited and calibrated using the Astronomical Image Processing System (AIPS). The bandpass, delay, phase, and polarization calibration were performed on the calibrator J2202+4216. Fringe-fitting and self-calibration were performed on the brightest maser feature (M05 in Table 1). Then the $I, Q, U$, and $V$ cubes were imaged ( $\left.\mathrm{rms}=2.4 \mathrm{mJy} \mathrm{beam}^{-1}\right)$ using the AIPS task IMAGR. The beam size was 7.47 mas $\times 3.38$ mas $\left(\mathrm{PA}=76^{\circ}\right)$. The $Q$ and $U$ cubes were combined to produce cubes of polarized intensity $\left(P O L I=\sqrt{Q^{2}+U^{2}}\right)$ and polarization angle $(\chi=1 / 2 \times \operatorname{atan}(U / Q))$. We calibrated the linear polarization angles by comparing the linear polarization angle of the polarization calibrator measured by us with the angle obtained by calibrating the POLCAL observations made by $\mathrm{NRAO}^{2}$. IRAS 20126+4104 was observed between two POLCAL observations runs during which the linear polarization angle of $\mathrm{J} 2202+4216$ was constant, with an average value of $-31^{\circ} \pm 1^{\circ}$. We were therefore able to estimate the polarization angle with a systemic error of no more than $\sim 1^{\circ}$. The formal errors on $\chi$ are due to thermal noise. This error is given by $\sigma_{\chi}=0.5 \sigma_{P} / P \times$ $180^{\circ} / \pi$ (Wardle \& Kronberg 1974), where $P$ and $\sigma_{P}$ are the polarization intensity and corresponding rms error, respectively.

2 http://www.aoc.nrao.edu/ smyers/calibration/ 
Table 2. Parameters of the 22-GHz $\mathrm{H}_{2} \mathrm{O}$ maser features detected in IRAS 20126+4104.

\begin{tabular}{ccccccccccccc}
\hline \hline $\begin{array}{c}(1) \\
\text { Maser }\end{array}$ & $\begin{array}{c}(2) \\
\mathrm{RA}^{a} \\
\text { offset } \\
(\mathrm{mas})\end{array}$ & $\begin{array}{c}(3) \\
\mathrm{Dec}^{a} \\
\text { offset } \\
(\mathrm{mas})\end{array}$ & $\begin{array}{c}\text { Peak flux } \\
\text { density(I) } \\
(\mathrm{Jy} / \mathrm{beam})\end{array}$ & $\begin{array}{c}V_{\mathrm{lsr}} \\
\left(\mathrm{km} \mathrm{s}^{-1}\right)\end{array}$ & $\begin{array}{c}\Delta v_{\mathrm{L}} \\
\left(\mathrm{km} \mathrm{s}^{-1}\right)\end{array}$ & $\begin{array}{c}(7) \\
P_{\mathrm{l}}{ }^{b}\end{array}$ & $\begin{array}{c}(8) \\
\chi^{b}\end{array}$ & $\begin{array}{c}(9) \\
\Delta V_{\mathrm{i}}{ }^{c}\end{array}$ & $\begin{array}{c}(10) \\
T_{B} \Delta \Omega^{c}\end{array}$ & $\begin{array}{c}(11) \\
P_{V}\end{array}$ & $\begin{array}{c}(12) \\
\Delta V_{\mathrm{Z}}\end{array}$ & $\begin{array}{c}(13) \\
\theta^{d}\end{array}$ \\
\hline W01 & -0.818 & -0.656 & $0.37 \pm 0.05$ & -2.05 & 0.74 & - & - & - & - & - & - & - \\
W02 & 0 & 0 & $24.77 \pm 0.06$ & -4.61 & 1.37 & $1.3 \pm 0.2$ & $-37 \pm 13$ & $<0.5$ & $9.1_{-0.4}^{+0.3}$ & - & - & $90_{-9}^{+9}$ \\
W03 & 403.317 & -212.020 & $0.19 \pm 0.04$ & -5.61 & 0.73 & - & - & - & - & - & - & - \\
W04 & 403.898 & -212.452 & $0.39 \pm 0.05$ & -6.23 & 0.55 & - & - & - & - & - & - & - \\
W05 & 542.648 & -201.458 & $0.23 \pm 0.05$ & -15.51 & 0.39 & - & - & - & - & - & - & - \\
\hline
\end{tabular}

Notes. ${ }^{(a)}$ The reference position is $\alpha_{2000}=20^{\mathrm{h}} 14^{\mathrm{m}} 25^{\mathrm{s}} .966 \pm 0^{\mathrm{s}} .002$ and $\delta_{2000}=41^{\circ} 13^{\prime} 32^{\prime \prime} .738 \pm 0^{\prime \prime} .014$ (see Sect. 4). ${ }^{(b)} P_{1}$ and $\chi$ are the mean values of the linear polarization fraction and the linear polarization angle measured across the spectrum, respectively. ${ }^{(c)}$ The best-fitting results obtained by using a model based on the radiative transfer theory of $\mathrm{H}_{2} \mathrm{O}$ masers for $\Gamma+\Gamma_{v}=1 \mathrm{~s}^{-1}$ (Surcis et al. 2011b). The errors were determined by analyzing the full probability distribution function. ${ }^{(d)}$ The angle between the magnetic field and the maser propagation direction is determined by using the observed $P_{1}$ and the fitted emerging brightness temperature. The errors were determined by analyzing the full probability distribution function.

\subsection{2-GHz VLBA data}

The star-forming region was also observed in the $6_{16}-5_{23}$ transition of $\mathrm{H}_{2} \mathrm{O}$ (rest frequency: $22.23508 \mathrm{GHz}$ ) with the $\mathrm{NRAO}^{3}$ VLBA on June 24, 2012. The observations were made in full polarization mode using a bandwidth of $4 \mathrm{MHz}$ to cover a velocity range of $\sim 54 \mathrm{~km} \mathrm{~s}^{-1}$. The data were correlated with the DiFX correlator using 2000 channels and generating all four polarization combinations (RR, LL, RL, LR) with a spectral resolution of $2 \mathrm{kHz}\left(\sim 0.03 \mathrm{~km} \mathrm{~s}^{-1}\right)$. Including the overheads, the total observation time was $8 \mathrm{~h}$.

The data were edited and calibrated using AIPS following the method of Kemball et al. (1995). The bandpass, the delay, the phase, and the polarization calibration were performed on the calibrator $\mathbf{J} 2202+4216$. The fringe-fitting and the self-calibration were performed on the brightest maser feature (W02 in Table 2). Then we imaged the $I, Q, U$, and $V$ cubes $\left(\mathrm{rms}=20 \mathrm{mJy} \mathrm{beam}^{-1}\right)$ using the AIPS task IMAGR (beam size 0.75 mas $\times 0.34$ mas, $\left.\mathrm{PA}=-9.4^{\circ}\right)$. The $Q$ and $U$ cubes were combined to produce cubes of POLI and $\chi$. Because IRAS 20126+4104 was observed ten days before a POLCAL observations run, we calibrated the linear polarization angles of the $\mathrm{H}_{2} \mathrm{O}$ masers by comparing the linear polarization angle of J2202+4216 measured by us with the angles measured during that POLCAL observations run $\left(\chi_{\mathrm{J} 2202+4216}=-15^{\circ} .0 \pm 0.3\right)$. Also in the case of the $\mathrm{H}_{2} \mathrm{O}$ masers, the $\sigma_{\chi}$ is due to thermal noise.

\section{Analysis}

The $\mathrm{CH}_{3} \mathrm{OH}$ and $\mathrm{H}_{2} \mathrm{O}$ maser features were identified by using the process described in Surcis et al. (2011b). We determined the mean linear polarization fraction $\left(P_{1}\right)$ and the mean linear polarization angle $(\chi)$ of each $\mathrm{CH}_{3} \mathrm{OH}$ and $\mathrm{H}_{2} \mathrm{O}$ maser feature by only considering the consecutive channels (more than two) across the total intensity spectrum for which $P O L I \geq 5 \sigma$.

We fitted the total intensity and the linearly polarized spectra of $\mathrm{H}_{2} \mathrm{O}$ and $\mathrm{CH}_{3} \mathrm{OH}$ maser features, for which we were able to detect linearly polarized emission, by using the full radiative transfer method (FRTM) code for $22-\mathrm{GHz} \mathrm{H}_{2} \mathrm{O}$ masers

\footnotetext{
3 The National Radio Astronomy Observatory (NRAO) is a facility of the National Science Foundation operated under cooperative agreement by Associated Universities, Inc.
}

(Vlemmings et al. 2006; Surcis et al. 2011b) and the adapted version of the code for 6.7- $\mathrm{GHz} \mathrm{CH} \mathrm{CH}_{3} \mathrm{OH}$ masers (Vlemmings et al. 2010; Surcis et al. 2011a). The code is based on the models of Nedoluha \& Watson (1992), who solved the transfer equations for the polarized radiation of $22-\mathrm{GHz} \mathrm{H}_{2} \mathrm{O}$ masers in the presence of a magnetic field causing a Zeeman splitting $\left(\Delta V_{\mathrm{Z}}\right)$ that is much smaller than the spectral line breadth.

We modeled the observed spectra by gridding the intrinsic thermal linewidth $\left(\Delta V_{\mathrm{i}}\right)$ in the case of $\mathrm{H}_{2} \mathrm{O}$ masers from 0.5 to $3.5 \mathrm{~km} \mathrm{~s}^{-1}$ in steps of $0.025 \mathrm{~km} \mathrm{~s}^{-1}$, and in the case of the $\mathrm{CH}_{3} \mathrm{OH}$ masers from 0.5 to $2.4 \mathrm{~km} \mathrm{~s}^{-1}$ in steps of $0.05 \mathrm{~km} \mathrm{~s}^{-1}$, by using a least-square fitting routine. The output of the codes provides estimates of the emerging brightness temperature $\left(T_{B} \Delta \Omega\right)$ and of $\Delta V_{\mathrm{i}}$. From the fit results, we were able to determine the best estimates of the angle between the maser propagation direction and the magnetic field $(\theta)$, because both shape and strength of the linear polarization spectrum depend (nonlinearly) on the maser saturation level and $\theta$. If $\theta>\theta_{\text {crit }}=55^{\circ}$, where $\theta_{\text {crit }}$ is the Van Vleck angle, the magnetic field appears to be perpendicular to the linear polarization vectors; otherwise, it is parallel (Goldreich et al. 1973). To better determine the orientation of the magnetic field with respect to the linear polarization vectors, Surcis et al. (2013) introduced a method that takes the errors associated to $\theta$ into consideration (i.e., $\theta_{\varepsilon^{-}}^{\varepsilon^{+}}$in Tables 2 and 1). We state that if $\left|\theta^{+}-55^{\circ}\right|>\left|\theta^{-}-55^{\circ}\right|$, where $\theta^{ \pm}=\theta \pm \varepsilon^{ \pm}$, the magnetic field is most likely perpendicular to the linear polarization vectors; otherwise, the magnetic field is assumed to be parallel. Of course, if $\theta^{-}$and $\theta^{+}$are both larger or smaller than $55^{\circ}$ the magnetic field is perpendicular or parallel to the linear polarization vectors, respectively.

Moreover, the best estimates for $T_{B} \Delta \Omega$ and $\Delta V_{\mathrm{i}}$ are included in the corresponding code to produce the $I$ and $V$ models that were used for fitting the total intensity and circular polarized spectra of the corresponding maser feature.

\section{Results}

Tables 1 and 2 list the 26 6.7- $\mathrm{GHz} \mathrm{CH}_{3} \mathrm{OH}$ maser features (named M01-M26) and the 5 22-GHz $\mathrm{H}_{2} \mathrm{O}$ maser features (named W01-W05), respectively, that we detected towards IRAS 20126+4104. They are all shown in Fig. 1. Because we did not observe in phase-referencing mode, we do not have information for the absolute position of both maser species. Still, 

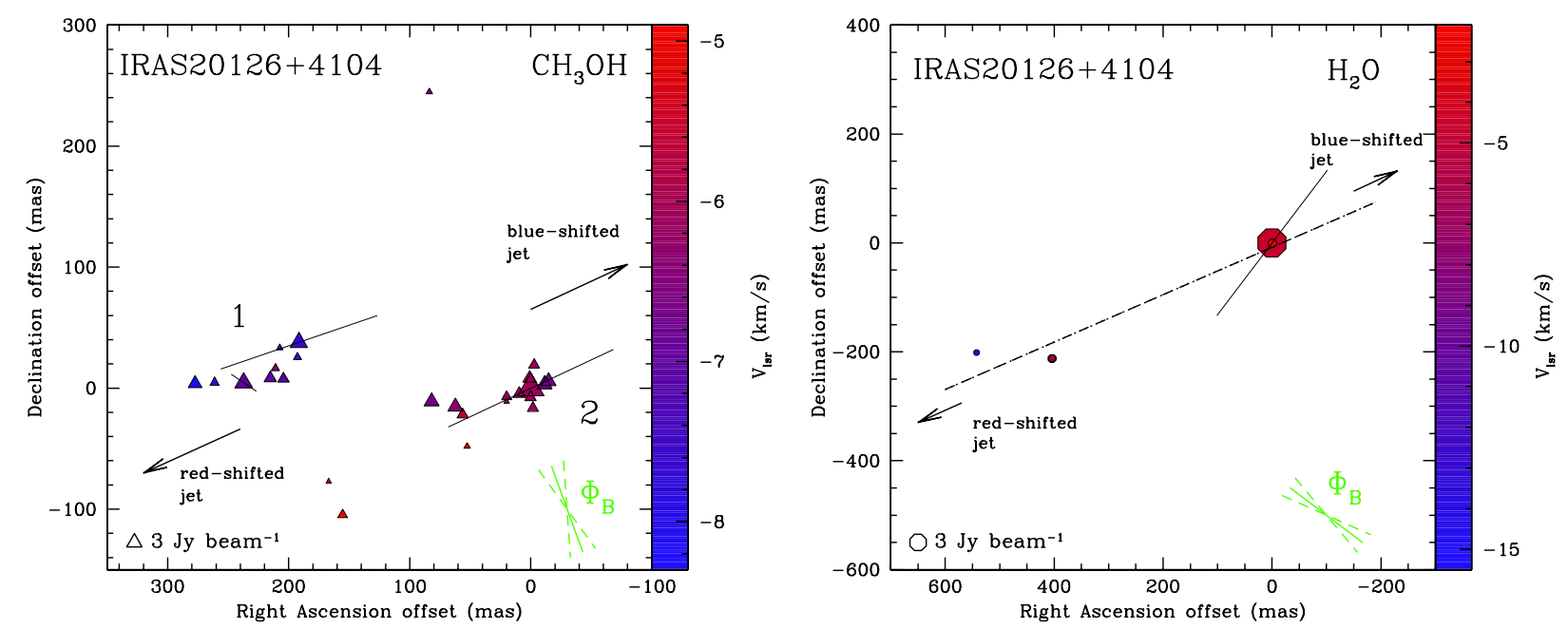

Fig. 1. Left panel: a view of the 6.7- $\mathrm{GHz} \mathrm{CH}_{3} \mathrm{OH}$ maser features detected around IRAS 20126+4104, the reference position is $\alpha_{2000}=$ $20^{\mathrm{h}} 14^{\mathrm{m}} 26^{\mathrm{s}} .046$ and $\delta_{2000}=41^{\circ} 13^{\prime} 32^{\prime \prime} .690$ (see Sect. 4). Right panel: a view of the 22-GHz $\mathrm{H}_{2} \mathrm{O}$ maser features detected around IRAS 20126+4104, the reference position is $\alpha_{2000}=20^{\mathrm{h}} 14^{\mathrm{m}} 25^{\mathrm{s}} .966$ and $\delta_{2000}=41^{\circ} 13^{\prime} 32^{\prime \prime} .738$ (see Sect. 4). The triangles and the octagonal symbols are the identified $\mathrm{CH}_{3} \mathrm{OH}$ and $\mathrm{H}_{2} \mathrm{O}$ maser features, respectively, scaled logarithmically according to their peak flux density (Tables 1 and 2). The maser LSR radial velocity is indicated by color. (The systemic velocity of IRAS $20126+4104$ is $V_{\mathrm{lsr}}=-3.5 \mathrm{~km} \mathrm{~s}^{-1}$, MCR11.) A $3 \mathrm{Jy}_{\text {beam }}{ }^{-1}$ symbol is plotted for illustration in both panels. The linear polarization vectors, scaled logarithmically according to polarization fraction $P_{1}$, are overplotted. In the right bottom corner of both panels, the error-weighted orientation of the magnetic field $\left(\Phi_{B}\right.$, see Sect. 5.3) is also reported, the two dashed segments indicate the uncertainties. The two arrows indicate the direction but not the absolute position of the red- and blue-shifted lobes of the jet $\left(\mathrm{PA}_{j \mathrm{jet}}=115^{\circ}\right.$; MCR11). The dotted line is the best linear fit of the $\mathrm{H}_{2} \mathrm{O}$ maser features $\left(\mathrm{PA}_{\mathrm{H}_{2} \mathrm{O}}=114^{\circ} \pm 4^{\circ}\right)$.

we were able to estimate the absolute position of the brightest features of both maser species (M05 and W02) through fringe rate mapping using the AIPS task FRMAP. The absolute position errors are $\Delta \alpha^{\mathrm{M} 05}=6$ mas and $\Delta \delta^{\mathrm{M} 05}=9$ mas for the $\mathrm{CH}_{3} \mathrm{OH}$ maser feature, and $\Delta \alpha^{\mathrm{W} 02}=24$ mas and $\Delta \delta^{\mathrm{W} 02}=$ 14 mas for the $\mathrm{H}_{2} \mathrm{O}$ maser feature. The position of the brightest $\mathrm{CH}_{3} \mathrm{OH}$ maser feature M05, which is Feature 1 in MCR11, agrees within $2 \sigma$ with the position of Feature 1 after considering the change in position due to the proper motion of the $\mathrm{CH}_{3} \mathrm{OH}$ masers (-4 mas $\mathrm{yr}^{-1}$ both in RA and in Dec, MCR11).

The description of the maser distribution and the polarization results are reported for each maser species separately below.

\section{1. $\mathrm{CH}_{3} \mathrm{OH}$ masers}

The $\mathrm{CH}_{3} \mathrm{OH}$ maser features can be divided into two groups, 1 and 2, following the naming convention of MCR11. An additional maser feature M15, which is undetected by MCR11, is about 200 mas north from the other maser features and cannot be included in any of these two groups. The spatial distribution and the velocity ranges of the two groups are consistent with those of MCR11.

We detected linear polarization in three $\mathrm{CH}_{3} \mathrm{OH}$ maser features $\left(P_{1}=0.6 \%-1.6 \%\right.$, see Fig. 2$)$, and the error-weighted linear polarization angles is $\langle\chi\rangle_{\mathrm{CH}_{3} \mathrm{OH}}=-70^{\circ} \pm 16^{\circ}$. The adapted version of the FRTM code was able to properly fit all these three $\mathrm{CH}_{3} \mathrm{OH}$ maser features, and the outputs with their relative errors are reported in Cols. 10, 11, and 14 of Table 1. Moreover, these maser features appear to be unsaturated, because their $T_{B} \Delta \Omega$ are under the saturation threshold $\left(T_{B} \Delta \Omega\right)_{\mathrm{CH}_{3} \mathrm{OH}}=2.6 \times$ $10^{9} \mathrm{~K}$ sr of the 6.7-GHz CH $\mathrm{CH}_{3} \mathrm{OH}$ masers (Surcis et al. 2011a). Considering the determined $\theta$ angles, the magnetic field is perpendicular to the linear polarization vectors, i.e., $\left|\theta^{+}-55^{\circ}\right|>$ $\left|\theta^{-}-55^{\circ}\right|$. Furthermore, we detected circularly polarized emission $\left(P_{V}=0.6 \%\right)$ toward the brightest $\mathrm{CH}_{3} \mathrm{OH}$ maser feature
M05, for which we measured quite a large Zeeman splitting $\Delta V_{\mathrm{Z}}=(-9.2 \pm 1.4) \mathrm{m} \mathrm{s}^{-1}$.

\section{2. $\mathrm{H}_{2} \mathrm{O}$ masers}

The $\mathrm{H}_{2} \mathrm{O}$ maser features are linearly distributed $\left(\mathrm{PA}_{\mathrm{H}_{2} \mathrm{O}}=114^{\circ} \pm\right.$ $4^{\circ}$ ) from northwest (NW) to southeast (SE), and their velocities increase in magnitude from NW to SE. The velocity of W05, which is the most southeastern and the most blue-shifted $\mathrm{H}_{2} \mathrm{O}$ maser features, is an order of magnitude faster than the velocities of the other maser features. Although the $\mathrm{PA}_{\mathrm{H}_{2} \mathrm{O}}$ of the maser distribution agrees perfectly with the PA measured recently by MCR11, the maser features are not on the outflow as detected by MCR11 and the velocity distribution is reversed with respect to what MCR11 observed (see Fig. 4).

We detected linearly polarized emission $\left(P_{1}=1.3 \%\right.$, see Fig. 2) only from the brightest $\mathrm{H}_{2} \mathrm{O}$ maser feature $\mathrm{W} 02$ $\left(\chi=-37^{\circ} \pm 13^{\circ}\right)$. The FRTM code provides an upper limit of $\Delta V_{\mathrm{i}}$ (Col. 9 of Table 2), while the value of $T_{B} \Delta \Omega$ (Col. 10) is below the saturation threshold $\left(T_{B} \Delta \Omega\right)_{\mathrm{H}_{2} \mathrm{O}}=6.7 \times 10^{9} \mathrm{~K} \mathrm{sr}$ also for the $\mathrm{H}_{2} \mathrm{O}$ maser, indicating an unsaturated maser (Surcis et al. 2011a). The third output of the FRTM code, i.e. $\theta$ (Col. 13), indicates that the magnetic field is on the plane of the sky and perpendicular to the linear polarization vector. No circular polarization at $5 \sigma$ was detected toward any $\mathrm{H}_{2} \mathrm{O}$ maser feature $\left(P_{\mathrm{V}}^{\mathrm{W} 02}<0.4 \%\right)$

\section{Discussion}

\subsection{Zeeman splitting}

The magnetic field strength along the line of sight can be calculated from the Zeeman-splitting measurements by using

$B_{\|}=\frac{\Delta V_{\mathrm{Z}}}{\alpha_{\mathrm{Z}}}$ 

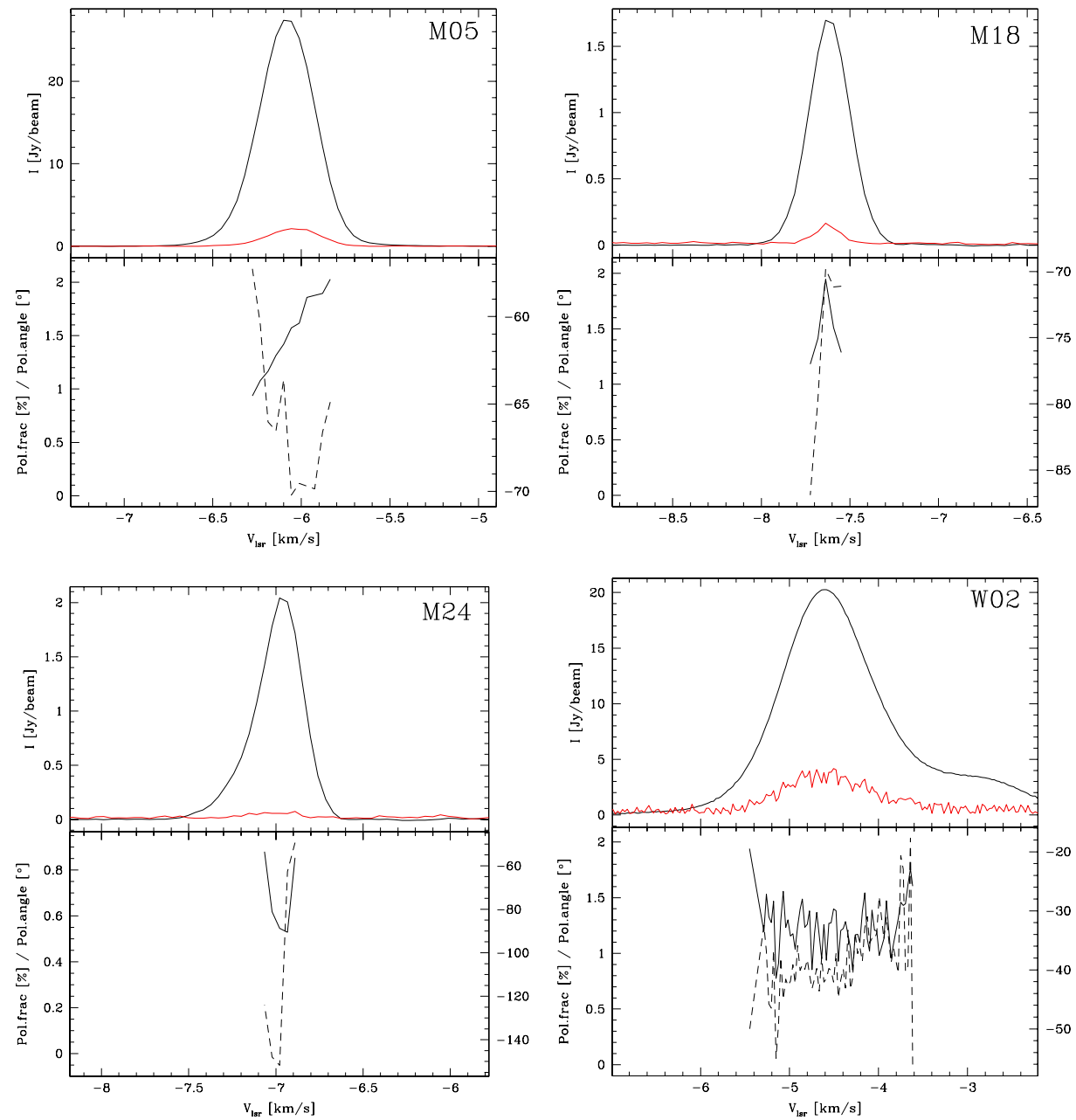

Fig. 2. Total intensity ( $I$, black solid line) and linear polarization intensity (red solid line) spectra of the $\mathrm{CH}_{3} \mathrm{OH}$ maser features $\mathrm{M} 05$, $\mathrm{M} 18$, and $\mathrm{M} 24$, and of the $\mathrm{H}_{2} \mathrm{O}$ maser feature W02 (upper panel). The linear polarization intensity spectra have been multiplied by a factor of five for the M05, M18, and M24, and by a factor of fifteen for W02. The spectra of polarization fraction (black solid line, left scale) and polarization angle (dashed black line, right scale) are also shown (lower panel).

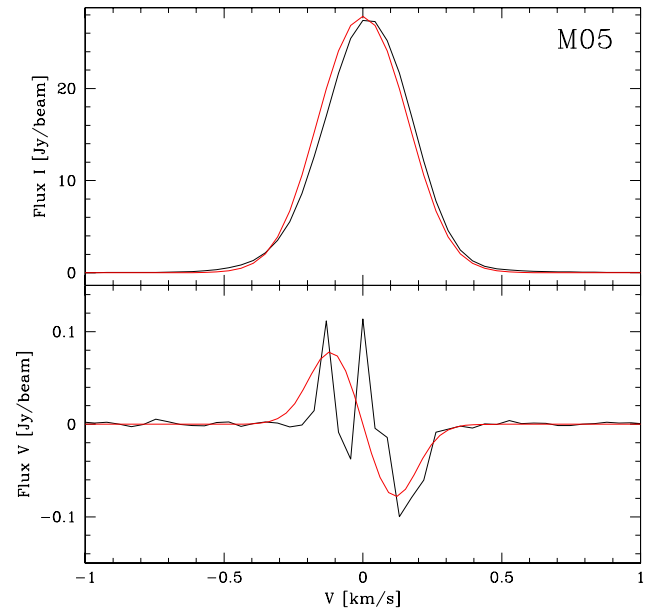

Fig. 3. Total intensity (I, upper panel) and circular polarization intensity ( $V$, lower panel) spectra for the $\mathrm{CH}_{3} \mathrm{OH}$ maser feature M05. The thick red line shows the best-fit models of $I$ and $V$ emission obtained using the adapted FRTM code (see Sect. 3). The maser features were centered on zero velocity.

where $\alpha_{Z}$ is the Zeeman-splitting coefficient, which depends on the Landé g-factor of the corresponding maser transition. Moreover, the total magnetic field strength can be determined if the angle between the maser propagation direction and the magnetic field $\theta$ is known, i.e., $B=B_{\|} / \cos \theta$. While the Zeeman-splitting coefficient for the $22-\mathrm{GHz} \mathrm{H}_{2} \mathrm{O}$ maser is well-known, $\alpha_{\mathrm{Z}}$ for the $6.7-\mathrm{GHz} \mathrm{CH}_{3} \mathrm{OH}$ maser emission is still uncertain. Indeed, the Landé g-factor corresponding to the $\mathrm{CH}_{3} \mathrm{OH}$ maser transition is still unknown (Vlemmings et al. 2011). However, a considerable value of $\alpha_{\mathrm{Z}}^{\mathrm{CH}_{3} \mathrm{OH}}$ could be in the range $0.005 \mathrm{~km} \mathrm{~s}^{-1} \mathrm{G}^{-1}<\alpha_{\mathrm{Z}}^{\mathrm{CH}_{3} \mathrm{OH}}<0.05 \mathrm{~km} \mathrm{~s}^{-1} \mathrm{G}^{-1}$ (Surcis et al. 2011a).

From our observations we measured Zeeman splitting only from the $\mathrm{CH}_{3} \mathrm{OH}$ maser M05, and consequently we can speculatively give only a possible range of $B_{\|}$, which is $0.2 \mathrm{G}<$ $\left|B_{\|}^{\mathrm{CH}_{3} \mathrm{OH}}\right|<2.1 \mathrm{G}$ where the uncertainty of $\Delta V_{\mathrm{Z}}$ has been taken into account. Considering $\theta_{\mathrm{M} 05}=75^{\circ}+40^{\circ}$, the total magnetic field, $B_{\mathrm{CH}_{3} \mathrm{OH}}$, ranges from $-0.2 \mathrm{G}$ to $-24 \mathrm{G}$. According to the sign of the Zeeman splitting, the magnetic field is pointing toward the observer. The non-detection of significant circular polarized emission from the $22-\mathrm{GHz}_{2} \mathrm{O}$ maser could be due to a weaker magnetic field along the outflows.

\subsection{Faraday rotation}

The interstellar medium (ISM) between IRAS 20126+4104 and the observer causes a rotation of the linear polarization vectors known as foreground Faraday rotation $\left(\Phi_{\mathrm{f}}\right)$. Even if previous works (e.g., Surcis et al. 2011a, 2012, 2013) have shown that this rotation is small at both $6.7-\mathrm{GHz}$ and $22-\mathrm{GHz}$ and do not affect the measurements of the magnetic field orientation, it is important to determine $\Phi_{\mathrm{f}}$ for IRAS $20126+4104$. The foreground 

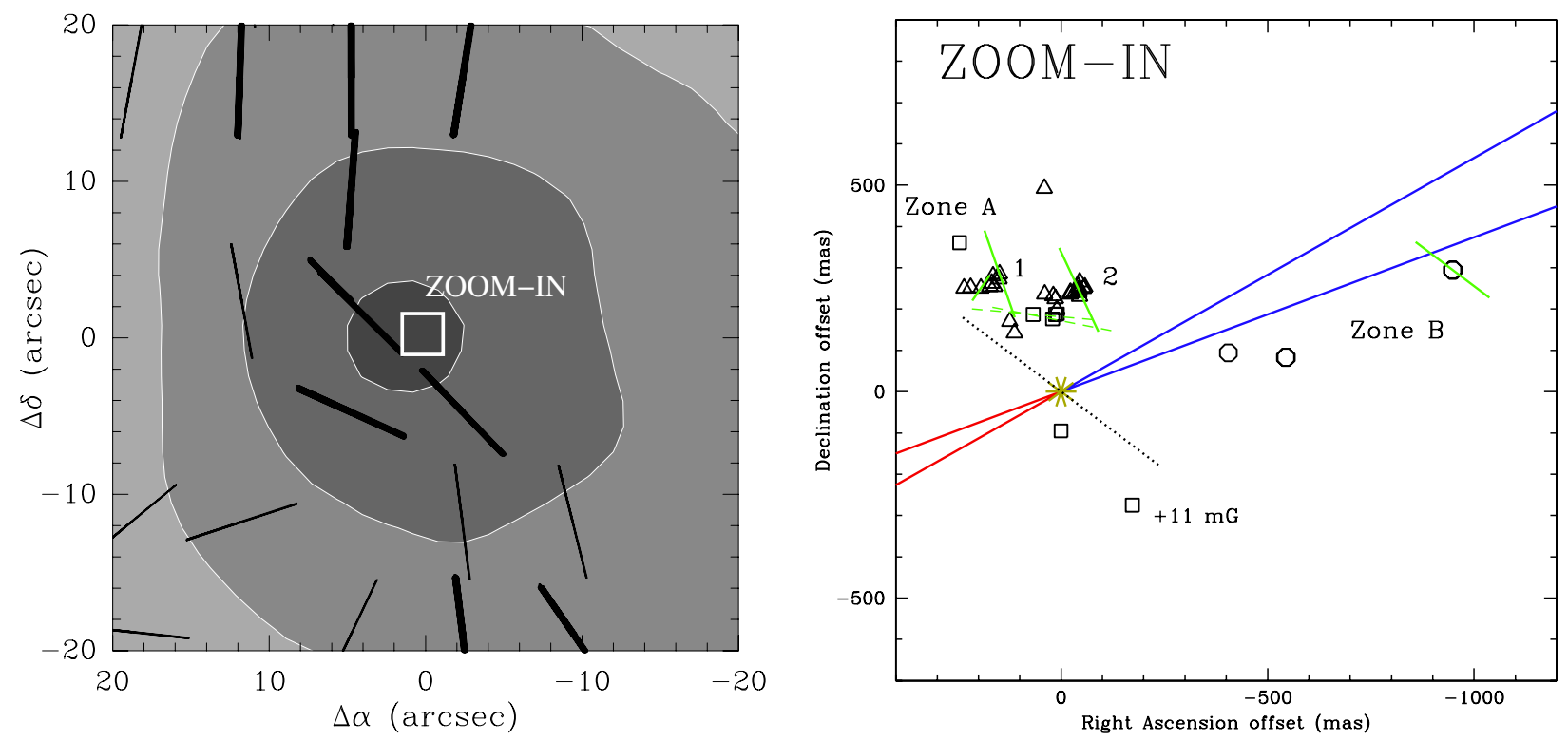

Fig. 4. Left panel: modified version of Fig. 3(b) of Shinnaga et al. (2012). The white box indicates the position of the right panel. The black bars represent the magnetic field direction determined from the polarized dust emission at $350 \mu \mathrm{m}$, whose continuum emission is in the background. Right panel: $\mathrm{CH}_{3} \mathrm{OH}$ (triangles), $\mathrm{OH}$ (squares) (Edris et al. 2005), and $\mathrm{H}_{2} \mathrm{O}$ (octagons) masers in IRAS 20126+4104. The gold asterisk represents the B0.5 protostar $\left(\alpha_{2000}=20^{\mathrm{h}} 14^{\mathrm{m}} 26^{\mathrm{s}} .0498\right.$ and $\delta_{2000}=41^{\circ} 13^{\prime} 32^{\prime \prime} .443$, MCR11), while the dotted line represents the Keplerian disk of $\sim 1000$ au $\left(\mathrm{PA}_{\text {disk }}=53^{\circ} \pm 7^{\circ}\right.$, Cesaroni et al. 2005). The red and blue lines indicate the red- and blue-shifted lobes of the jet, respectively, with a $\mathrm{PA}_{\text {jet }}=$ $115^{\circ}$ and an opening angle of $9^{\circ}$ (MCR11). The thick green segments represent the magnetic field direction determined from the polarized $\mathrm{CH}_{3} \mathrm{OH}$ and $\mathrm{H}_{2} \mathrm{O}$ maser emissions. The green dashed segments represent the magnetic field direction determined from the linearly polarized emission of $\mathrm{OH}$ masers (Edris et al. 2005). The foreground Faraday rotation at 1.6-GHz is probably not negligible and needs to be taken into account when interpreting the image (see Sect. 5.2).

Faraday rotation is given by

$\Phi_{\mathrm{f}}\left[^{\circ}\right]=4.22 \times 10^{6}\left(\frac{D}{[\mathrm{kpc}]}\right)\left(\frac{n_{\mathrm{e}}}{\left[\mathrm{cm}^{-3}\right]}\right)\left(\frac{B_{\|}}{[\mathrm{mG}]}\right)\left(\frac{v}{[\mathrm{GHz}]}\right)^{-2}$,

where $D$ is the length of the path over which the Faraday rotation occurs, $n_{\mathrm{e}}$ and $B_{\|}$are the average electron density and the magnetic field along this path, respectively, and $v$ is the frequency. By assuming that the interstellar electron density, magnetic field, and distance are $n_{\mathrm{e}} \approx 0.012 \mathrm{~cm}^{-3}, B_{\|} \approx 2 \mu \mathrm{G}$ (Sun et al. 2008), and $D=1.64 \mathrm{kpc}$, respectively, $\Phi_{\mathrm{f}}$ is estimated to be 4.0 at $6.7-\mathrm{GHz}$ and 0.3 at $22-\mathrm{GHz}$, but for $1.6-\mathrm{GHz} \mathrm{OH}$ masers $\Phi_{\mathrm{f}} \approx 60^{\circ}$.

Surcis et al. $(2012,2013)$ found that the linear polarization vectors of $6.7-\mathrm{GHz} \mathrm{CH}_{3} \mathrm{OH}$ masers are quite accurately aligned in all the young stellar objects (YSOs) that they observed, indicating that the internal Faraday rotation $\left(\Phi_{i}\right)$ is negligible. In the case of 22- $\mathrm{GHz} \mathrm{H} \mathrm{H}_{2} \mathrm{O}$ masers, $\Phi_{\mathrm{i}}$ is found to be negligible only if the $\mathrm{H}_{2} \mathrm{O}$ masers are pumped by a C-shock (Kaufman \& Neufeld 1996).

\subsection{Morphology of the magnetic field}

The two maser species that are associated with two different structures of the YSO (i.e., the disk and the outflows, see Sect. 4) probe the morphology of the magnetic field in two different zones of the protostar. The magnetic field close to the disk (Zone A, at $\sim 400$ au from the protostar), which is probed by the $\mathrm{CH}_{3} \mathrm{OH}$ masers, has an orientation on the plane of the sky of $\Phi_{B}^{\text {disk }}=20^{\circ} \pm 16^{\circ}$, while close to the jet (Zone $\mathrm{B}$, at $\sim 1600$ au from the protostar), which is probed by the $\mathrm{H}_{2} \mathrm{O}$ masers, $\Phi_{B}^{\text {outflow }}=53^{\circ} \pm 13^{\circ}$ (see Fig. 4). A comparison of the morphology of the magnetic field with the structure of the protostar reveals that the magnetic field is parallel to the disk $\left(\mathrm{PA}_{\text {disk }}=53^{\circ} \pm 7^{\circ}\right.$; Cesaroni et al. 2006) in Zone B, and it rotates clockwise by $33^{\circ}$ in Zone A, i.e., at $\sim 400$ au from the central protostar. Here the magnetic field is perpendicular to the jet $\left(\mathrm{PA}_{\text {jet }}=115^{\circ} ; \mathrm{MCR} 11\right)$. Moreover, the angle between the magnetic field and the line of sight is $\langle\theta\rangle_{\mathrm{CH}_{3} \mathrm{OH}}=82_{-41^{\circ}}^{\circ+8^{\circ}}$ in Zone A and $\langle\theta\rangle_{\mathrm{H}_{2} \mathrm{O}}=90_{-9^{\circ}}^{\circ}$ in Zone B; i.e., the magnetic field is on the plane of the sky. Even if the magnetic field is not parallel to the jet, $\langle\theta\rangle_{\mathrm{CH}_{3} \mathrm{OH}}$ is consistent with the inclination of the jet with respect to the line of sight, which is $\varphi=80^{\circ}$ (MCR11). In addition, because $\Delta V_{\mathrm{Z}}$ is negative, the magnetic field in Zone $\mathrm{A}$ is pointing towards the observer (e.g., Surcis et al. 2011b). We note that Edris et al. (2005) identified one Zeeman pair of OH masers, which indicates a magnetic field strength of about $+11 \mathrm{mG}$ in the direction pointing away from the observer at the opposite side of the disk from Zone A (see Fig. 4). Therefore, this could be evidence for the reversal of the magnetic field from above to below the disk.

Shinnaga et al. (2012) measured an $S$-shaped morphology of the magnetic field on a large scale by observing the polarized dust emission at $350 \mu \mathrm{m}$ (see Fig. 4; angular resolution 9", which at $1.64 \mathrm{kpc}$ corresponds to $\sim 15000 \mathrm{au}$ ). They determined that the magnetic field changes its direction from N-S to E-W inside the infalling region $(r<0.1 \mathrm{pc} \approx 20000 \mathrm{AU})$. The orientation of the magnetic field determined from the linearly polarized emission of $\mathrm{CH}_{3} \mathrm{OH}$ and $\mathrm{H}_{2} \mathrm{O}$ masers is in good agreement with the large-scale magnetic field. The orientation of the magnetic field measured from the $\mathrm{OH}$ masers by Edris et al. (2005) suffers from a large uncertainty due to the large foreground Faraday rotation. Because the $\mathrm{OH}$ masers arise in the same projected area of the $\mathrm{CH}_{3} \mathrm{OH}$ masers, for which $\Phi_{\mathrm{f}}$ is small, the orientation of the magnetic field measured from both maser species could be expected to be the same. This implies that the magnetic field 
vectors of $\mathrm{OH}$ masers should be rotated of approximately $60^{\circ}$ to be consistent with those of the $\mathrm{CH}_{3} \mathrm{OH}$ masers. This rotation is equal to the foreground Faraday rotation estimated in Sect. 5.2. Consequently, the magnetic field derived from the $\mathrm{OH}$ maser emission would also be consistent with the $S$-shaped morphology measured by Shinnaga et al. (2012).

The good agreement of the magnetic field from small to large scale suggests that the $\mathrm{CH}_{3} \mathrm{OH}$ masers of Group 1 are not on the disk but they are likely to be tracing material that is being accreted onto the disk along the magnetic field line as in Cepheus A (Vlemmings et al. 2010). Indeed, if the $\mathrm{CH}_{3} \mathrm{OH}$ masers of Group 1 were on the disk, we would have expected a resulting magnetic field that is much more random because of turbulent motions in the disk (Seifried et al. 2012b). The $\mathrm{CH}_{3} \mathrm{OH}$ masers of Group 2 are instead interpreted as tracing the material in the disk winds that is flowing out along the twisted magnetic field lines. In this case, the $\mathrm{CH}_{3} \mathrm{OH}$ masers should have a helical motion, like the $\mathrm{SiO}$ masers in Orion (Matthews et al. 2010), which is consistent with the proper motion of Group 1 measured by MCR11.

\subsection{Role of the magnetic field}

To investigate the $S$-shaped morphology Shinnaga et al. (2012) calculated the evolution of a magnetized cloud that has the same observed parameters of IRAS $20126+4104$. They considered a constant magnetic field strength of $1.5 \times 10^{-5} \mathrm{G}$ parallel to the $z$ axis and with the rotation axis, which is rotated at an angle of $60^{\circ}$ with respect to the $z$ axis, on the $y-z$ plane. In their simulations the initial cloud has the energy ratios $E_{\text {rot }} / E_{\text {grav }}=0.02$ and $E_{B} / E_{\text {grav }}=0.55$, i.e. $E_{\text {rot }}<E_{B}$. Here $E_{\text {rot }}$ is the rotational energy, $E_{\text {grav }}$ the gravitational energy, and $E_{B}$ the magnetic energy in the cloud. They find that the simulated magnetic field vectors agree with the observed morphology of the magnetic field if the cloud is observed from the $x-y$ plane with a viewing angle of $30^{\circ}$ with respect to the $y$ axis. More recently, Kataoka et al. (2012) have shown that in star-forming cores the polarization distribution projected on the celestial plane strongly depends on the viewing angle of the cloud.

Kataoka et al. (2012) studied four different models in which they adopted a uniform magnetic field that has the same direction but different strengths for each model. In Models 3 and 4, the rotation of the cloud is introduced and the rotation axis is inclined from the magnetic field lines at an angle of $60^{\circ}$. Model 4 has the strongest magnetic field among all the models. According to their simulations, the large-scale $S$-shaped morphology, i.e. the magnetic field deviating from an hourglass configuration, in IRAS 20126+4104 might be explained by Model 3, and it is caused by (1) the misalignment of the magnetic field with the rotation axis and by (2) $E_{\mathrm{rot}}>E_{B}$. A slight misalignment of the magnetic field with the rotation axis was observed on a large scale by Shinnaga et al. (2012), who measured that the mean direction of the global magnetic field is $\Phi_{\mathrm{Bglobal}}=-3^{\circ}$, and the rotation axis of the cloud is $\mathrm{PA}_{\mathrm{rot}}=-40^{\circ} \pm 20^{\circ}$. Condition (2) of Kataoka et al. (2012) instead contradicts the initial conditions of the simulations made by Shinnaga et al. (2012).

So far, no observational determinations of the ratio between $E_{\text {rot }}$ and $E_{B}$ has been possible because no magnetic field strength has been measured in IRAS 20126+4104. But now we can determine if $E_{\text {rot }}>E_{B}$ (hereafter case A) or if $E_{\text {rot }}<E_{B}$ (hereafter case $\mathrm{B}$ ) by using our estimates of the magnetic field strength at $\mathrm{CH}_{3} \mathrm{OH}$ maser densities.

We assume that the cloud is a homogeneous solid sphere with magnetic flux freezing during its evolution. The rotational energy for a homogeneous solid sphere with radius $R$, mass $M$, and angular velocity $\Omega$ is

$E_{\mathrm{rot}}=\frac{1}{5} M R^{2} \Omega^{2}$

while the magnetic energy for the same sphere is

$E_{B}=\frac{1}{6}|B|^{2} R^{3}$,

where $|B|$ is the magnetic field strength into which the sphere is immersed. The critical value of magnetic field at which $E_{\mathrm{rot}}=$ $E_{B}$ is

$\left|B_{\text {critical }}\right|=\sqrt{\frac{6}{5}} \cdot \frac{M^{1 / 2} \Omega}{R^{1 / 2}}$.

Considering that the estimates for the cloud properties of IRAS $20126+4104$ are $R=0.54 \mathrm{pc}, M=402 M_{\odot}$ (Hofner et al. 2007), and $\Omega=2 \mathrm{~km} \mathrm{~s}^{-1} \mathrm{pc}^{-1}$ (Shinnaga et al. 2008), we find that the critical value of the magnetic field of the cloud should be

$\left|B_{\text {critical }}\right|=5 \times 10^{-5} \mathrm{G}$.

This $\left|B_{\text {critical }}\right|$ value is determined not at the $\mathrm{CH}_{3} \mathrm{OH}$ maser densities, so it cannot be directly compared with the magnetic field strength measured by us. But because we have assumed the presence of magnetic flux freezing in the cloud, the relation $|B| \propto n_{\mathrm{H}_{2}}^{\kappa}$, where $\kappa=0.47$ as empirically determined by Crutcher (1999), can be used to estimate $\left|B_{\text {critical }}\right|$ at the $\mathrm{CH}_{3} \mathrm{OH}$ maser densities. We assume $|B| \propto n_{\mathrm{H}_{2}}^{0.47}$ because it is proven to be valid up to densities of $10^{11} \mathrm{~cm}^{-3}$ (Vlemmings 2008). Cragg et al. (2005) determine that the number density of $6.7-\mathrm{GHz}$ $\mathrm{CH}_{3} \mathrm{OH}$ maser $\left(n_{\mathrm{H}_{2}}^{\mathrm{CH}_{3} \mathrm{OH}}\right)$ varies from $10^{7} \mathrm{~cm}^{-3}$ to $10^{9} \mathrm{~cm}^{-3}$, above which the $\mathrm{CH}_{3} \mathrm{OH}$ masers are quenched. Therefore, we have to estimate a range of $\left|B_{\text {critical }}\right|$ by considering the whole range of $n_{\mathrm{H}_{2}}^{\mathrm{CH}_{3} \mathrm{OH}}$. The critical value of the magnetic field at the densities of the $6.7-\mathrm{GHz} \mathrm{CH}_{3} \mathrm{OH}$ maser is thus between $\left|B_{\text {critical }}^{10^{7} \mathrm{~cm}^{-3}}\right|=0.001 \mathrm{G}$ and $\left|B_{\text {critical }}^{10^{9} \mathrm{~cm}^{-3}}\right|=0.01 \mathrm{G}$. Consequently, in Case A, $\left|B_{\text {critical }}^{10^{9} \mathrm{~cm}^{-3}}\right|_{\text {caseA }}<0.01 \mathrm{G}(\kappa=0.47)$, and in Case B, $\left|B_{\text {critical }}^{10^{7} \mathrm{~cm}^{-3}}\right|_{\text {caseB }}>0.001 \mathrm{G}(\kappa=0.47)$.

It is important to mention that Crutcher et al. (2010) claim a different value of $\kappa$, i.e. $\kappa=0.65$. They find that at densities less than $10^{2} \mathrm{~cm}^{-3}$, magnetic fields are density independent; i.e., they are constant, while for higher densities they vary as $|B| \propto n_{\mathrm{H}_{2}}^{0.65}$. Even though this relation has so far been verified for densities up to $10^{7} \mathrm{~cm}^{-3}$, for the sake of completeness we also estimate $\left|B_{\text {critical }}\right|$ at $\mathrm{CH}_{3} \mathrm{OH}$ maser densities by using $|B| \propto n_{\mathrm{H}_{2}}^{0.65}$. Repeating the calculation for $\kappa=0.65$, we found $\left|B_{\text {critical }}^{10^{9} \mathrm{~cm}^{-3}}\right|_{\text {caseA }}<0.1 \mathrm{G}(\kappa=0.65)$ and $\left|B_{\text {critical }}^{10^{7} \mathrm{~cm}^{-3}}\right|_{\text {caseB }}>0.004 \mathrm{G}$ $(\kappa=0.65)$.

In Fig. 5 we show a simple diagram that can help visualize the different $\left|B_{\text {critical }}\right|$ ranges and the measured $\left|B_{\mathrm{CH}_{3} \mathrm{OH}}\right|$, which are estimated by using both $\alpha_{\mathrm{Z}}=0.05 \mathrm{~km} \mathrm{~s}^{-1} \mathrm{G}^{-1}$ and $\alpha_{\mathrm{Z}}=0.005 \mathrm{~km} \mathrm{~s}^{-1} \mathrm{G}^{-1}$. To determine the ranges of $\left|B_{\mathrm{CH}_{3} \mathrm{OH}}\right|$, we also considered the errors of $\Delta V_{\mathrm{Z}}$ and $\theta$. We can see from Fig. 5 that the magnetic field measured from the Zeeman splitting of the $\mathrm{CH}_{3} \mathrm{OH}$ maser $\mathrm{M} 05$, independently of the value of $\alpha_{\mathrm{Z}}$ and $n_{\mathrm{H}_{2}}^{\mathrm{CH}_{3} \mathrm{OH}}$, indicates that $E_{\mathrm{rot}}<E_{B}$ (both for $\kappa=0.47$ and for $\kappa=0.65)$.

Using similar calculations for $1.6-\mathrm{GHz}$ OH maser $\left(10^{5} \mathrm{~cm}^{-3}<n_{\mathrm{H}_{2}}<10^{8} \mathrm{~cm}^{-3}\right.$; Crutcher 2012), we find 


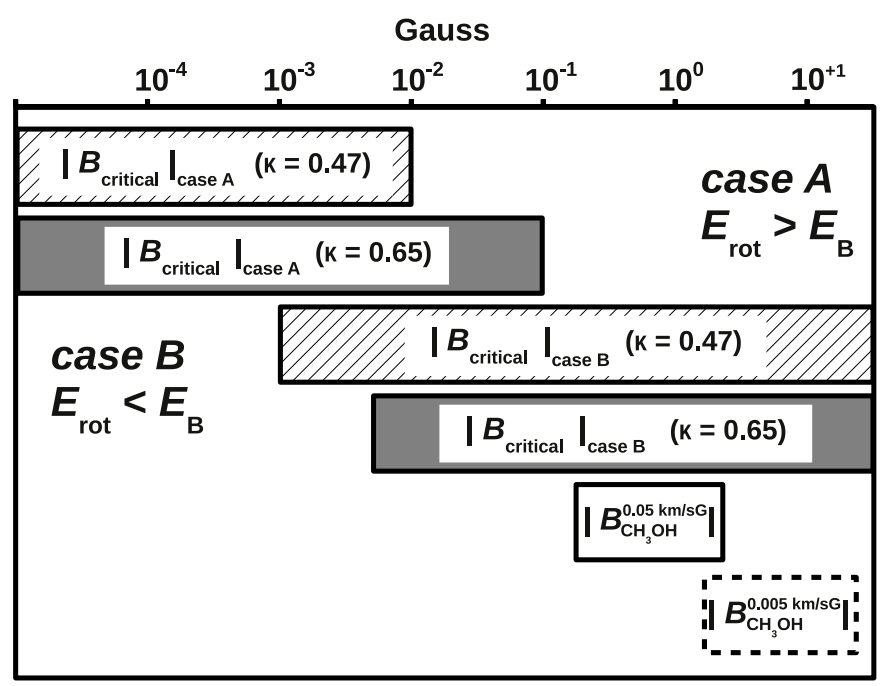

Fig. 5. A comparison between the magnetic field strength estimated from the Zeeman splitting of the $\mathrm{CH}_{3} \mathrm{OH}$ maser feature M05 and the critical magnetic field strength if $E_{\mathrm{rot}}>E_{B}$ and if $E_{\mathrm{rot}}<E_{B}$. The hatched area and the dark gray area show the critical magnetic field strength in case $\kappa=0.47$ (Crutcher 1999) and in case $\kappa=0.65$ (Crutcher et al. 2010), respectively. The full box indicates the range of $\left|B_{\mathrm{CH}_{3} \mathrm{OH}}\right|$ measured by considering $\alpha_{\mathrm{Z}}^{\mathrm{CH}_{3} \mathrm{OH}}=0.05 \mathrm{~km} \mathrm{~s}^{-1} \mathrm{G}^{-1}$, while the dashed box indicates the range of $\left|B_{\mathrm{CH}_{3} \mathrm{OH}}\right|$ if $\alpha_{\mathrm{Z}}^{\mathrm{CH}_{3} \mathrm{OH}}=0.005 \mathrm{~km} \mathrm{~s}^{-1} \mathrm{G}^{-1}$. The ranges are estimated considering both the errors of $\Delta V_{\mathrm{Z}}$ and $\theta$.

that the magnetic field strength measured by Edris et al. (2005), i.e. $11 \mathrm{mG}$, satisfies Case B, i.e. $E_{\text {rot }}<E_{B}$, only if $\kappa=0.47$ $\left(\left|B_{\text {critical }}^{10^{8} \mathrm{~cm}^{-3}}\right|_{\text {caseB }}>10^{-4} \mathrm{G}\right)$ and Case A, i.e. $E_{\text {rot }}>E_{B}$, only if $\kappa=0.65\left(\left|B_{\text {critical }}^{10^{5} \mathrm{~cm}^{-3}}\right|_{\text {case }}<0.02 \mathrm{G}\right)$.

Therefore, in our estimates the magnetic field dominates the rotation of the cloud. Moreover, we can speculatively state that the initial conditions of Shinnaga et al. (2012) are correct and that the $S$-shaped morphology of the magnetic field cannot be described by Model 3 of Kataoka et al. (2012). However, in Model 4 of Kataoka et al. (2012), the magnetic field is stronger, and we have the initial condition $E_{\mathrm{rot}}<E_{B}$. In this case they find that the deviation of the magnetic field lines from the hourglass configuration could only be observed very close to the protostar, i.e., where the magnetic field is probed by the 6.7- $\mathrm{GHz} \mathrm{CH}_{3} \mathrm{OH}$ masers. Of course, further observations, for instance of dust tracers in full polarization mode at mas resolution, could in future help clarify the role of the magnetic field in IRAS $20126+4104$.

\section{Conclusions}

The YSO IRAS $20126+4104$ has been observed in full polarization spectral mode at $6.7-\mathrm{GHz}$ with the EVN and at 22-GHz with the VLBA to detect linear and circular polarization emission from $\mathrm{CH}_{3} \mathrm{OH}$ and $\mathrm{H}_{2} \mathrm{O}$ masers, respectively. We detected 26 $\mathrm{CH}_{3} \mathrm{OH}$ masers and $5 \mathrm{H}_{2} \mathrm{O}$ masers at mas resolution. Linearly polarized emission was detected towards three $\mathrm{CH}_{3} \mathrm{OH}$ masers and one $\mathrm{H}_{2} \mathrm{O}$ maser that probed the magnetic field both close to the Keplerian disk and to the large-scale outflow. The orientation of the magnetic field derived from the masers agrees with the $S$-shaped morphology that was measured by Shinnaga et al. (2012) on a larger scale by using dust-polarized emission at $350 \mu \mathrm{m}$.

Moreover, we were able to measure a Zeeman splitting of $-9.2 \mathrm{~m} \mathrm{~s}^{-1}$ from the brightest $6.7-\mathrm{GHz} \mathrm{CH}_{3} \mathrm{OH}$ maser. From this measurement, we determined that the magnetic field energy dominates the rotation energy of the region; i.e., $E_{\mathrm{rot}}<E_{B}$.

Acknowledgements. We wish to thank an anonymous referee for making useful suggestions that have improved the paper. The EVN is a joint facility of European, Chinese, South African, and other radio astronomy institutes funded by their national research councils.

\section{References}

Caratti o Garatti, A., Froebrich, D., Eislöffel, J., et al. 2008, A\&A, 485, 137 Cesaroni, R., Felli, M., Testi, L., et al. 1997, A\&A, 325, 725

Cesaroni, R., Felli, M., Jenness, T., et al. 1999, A\&A, 345, 949

Cesaroni, R., Neri, R., Olmi, L., et al. 2005, A\&A, 434, 1039

Cesaroni, R., Galli, D., Lodato, G., et al. 2006, Nature, 444, 703

Cesaroni, R., Galli, D., Lodato, G., et al. 2007, in Protostars and Planets V, eds.

B. Reipurth, D. Lewitt, \& K. Keil (Tucson: Univ. of Arizona Press), 197 Cesaroni, R., Massi, F., Arcidiacono, C., et al. 2013, A\&A, 549, A146 Cragg, D. M., Sobolev, A. M., \& Godfrey, P. D. 2005, MNRAS, 360, 533 Crutcher, R. M. 1999, ApJ, 520, 706

Crutcher, R. M. 2012, ARA\&A, 50, 29

Crutcher, R. M., Wandelt, B., Heiles, C., et al. 2010, ApJ, 725, 466 Edris, K. A., Fuller, G. A., Cohen, R. J., et al. 2005, A\&A, 434, 213

Goldreich, P., Keeley, D. A., \& Kwan, J. Y., 1973, ApJ, 179, 111

Hofner, P., Cesaroni, R., Olmi, L., et al. 2007, A\&A, 465, 197

Johnston, K. G., Shepherd, D. S., Robitaille, T. P., et al. 2013, A\&A, 551, A43

Kataoka, A., Machida, M., \& Tomisaka, K. 2012, ApJ, 761, 40

Kaufman, M. J., \& Neufeld, D. A., 1996, ApJ, 456, 250

Kemball, A. J., Diamond, P. J., \& Cotton, W. D. 1995, A\&AS, 110, 383

Keto, E., \& Zhang, Q. 2010, MNRAS, 405, 102

Matthews, L. D., Greenhill, L. J., Goddi, C., et al. 2010, ApJ, 708, 80

Moscadelli, L., Cesaroni, R., \& Rioja, M. J. 2005, A\&A, 438, 889

Moscadelli, L., Cesaroni, R., Riojia, M. J., et al. 2011, A\&A, 526, A66 (MCR11)

Myers, A. T., McKee, C. F., Cunningham, A. J., et al. 2013, ApJ, 766, 97

Nedoluha, G. E., \& Watson, W. D., 1992, ApJ, 384, 185

Peters, T., Banerjee, R., Klessen, R. S., et al. 2011, ApJ, 729, 72

Seifried, D., Banerjee, R., Klessen, R. S., et al. 2011, MNRAS, 417, 1054

Seifried, D., Pudritz, R. E., Banerjee, R., et al. 2012a, MNRAS, 422, 347

Seifried, D., Banerjee, R., Pudritz, R. E., et al. 2012b, MNRAS, 423, L40

Shepherd, D. S., Yu, K. C., Bally, J., et al. 2000, ApJ, 535, 833

Shinnaga, H., Phillips, T. G., Furuya, R. S., et al. 2008, ApJ, 682, 1103

Shinnaga, H., Novak, G., Vaillancourt, J. E., et al. 2012, ApJ, 750, L29

Sun, X. H., Reich, W., Waelkens, A., et al. 2008, A\&A, 477, 573

Surcis, G., Vlemmings, W. H. T., Torres, R. M., et al. 2011a, A\&A, 533, A47

Surcis, G., Vlemmings, W. H. T., Curiel, S., et al. 2011b, A\&A, 527, A48

Surcis, G., Vlemmings, W. H. T., van Langevelde, H. J., et al. 2012, A\&A, 541, A47

Surcis, G., Vlemmings, W. H. T., van Langevelde, H. J., et al. 2013, A\&A, 556, A73

Tang, Y.-W., Ho, P. T. P., Koch, P. M., et al. 2009, ApJ, 700, 251

Vlemmings, W. H. T. 2008, A\&A, 484, 773

Vlemmings, W. H. T., Diamond, P. J., van Langevelde, H. J., et al. 2006, A\&A, 448,597

Vlemmings, W. H. T., Surcis, G., Torstensson, K. J. E., et al. 2010, MNRAS, 404, 134

Vlemmings, W. H. T., Torres, R. M., \& Dodson, R. 2011, A\&A, 529, A95

Wardle, J. F. C., \& Kronberg, P. P. 1974, ApJ, 194, 249 